\title{
The role of vimentin in the tumor marker Nup88-dependent multinucleated phenotype
}

\author{
Masaki Makise ${ }^{*}$ (D), Hideaki Nakamura and Akihiko Kuniyasu
}

\begin{abstract}
Background: Nucleoporin Nup88, a component of nuclear pore complexes, is known to be overexpressed in several types of tumor tissue. The overexpression of Nup88 has been reported to promote the early step of tumorigenesis by inducing multinuclei in both HeLa cells and a mouse model. However, the molecular basis of how Nup88 leads to a multinucleated phenotype remains unclear because of a lack of information concerning its binding partners. In this study, we characterize a novel interaction between Nup88 and vimentin. We also examine the involvement of vimentin in the Nup88-dependent multinucleated phenotype.

Methods: Cells overexpressing tagged versions of Nup88, vimentin and their truncations were used in this study. Coprecipitation and GST-pulldown assays were carried out to analyze protein-protein interactions. Vimentin knockdown by siRNA was performed to examine the functional role of the Nup88-vimentin interaction in cells. The phosphorylation status of vimentin was analyzed by immunoblotting using an antibody specific for its phosphorylation site.

Results: Vimentin was identified as a Nup88 interacting partner, although it did not bind to other nucleoporins, such as Nup50, Nup214, and Nup358, in HeLa cell lysates. The N-terminal 541 amino acid residues of Nup88 was found to be responsible for its interaction with vimentin. Recombinant GST-tagged Nup88 bound to recombinant vimentin in a GST-pulldown assay. Although overexpression of Nup88 in HeLa cells was observed mainly at the nuclear rim and in the cytoplasm, colocalization with vimentin was only partially detected at or around the nuclear rim. Disruption of the Nup88-vimentin interaction by vimentin specific siRNA transfection suppressed the Nup88-dependent multinucleated phenotype. An excess amount of Nup88 in cell lysates inhibited the dephosphorylation of a serine residue (Ser83) within the vimentin $\mathrm{N}$-terminal region even in the absence and presence of an exogenous phosphatase. The $\mathrm{N}$-terminal 96 amino acid residues of vimentin interacted with both full-length and the N-terminal 541 residues of Nup88.
\end{abstract}

Conclusions: Nup88 can affect the phosphorylation status of vimentin, which may contribute to the Nup88-dependent multinucleated phenotype through changing the organization of vimentin.

Keywords: Nup88, Nucleoporin, Vimentin, Cancer, Multinucleated phenotype, Kinases, Interaction

* Correspondence: makise@ph.sojo-u.ac.jp
Faculty of Pharmaceutical Sciences, Sojo University, 4-22-1 Ikeda, Nishi-ku,

Kumamoto 860-0082, Japan

(c) The Author(s). 2018 Open Access This article is distributed under the terms of the Creative Commons Attribution 4.0 International License (http://creativecommons.org/licenses/by/4.0/), which permits unrestricted use, distribution, and reproduction in any medium, provided you give appropriate credit to the original author(s) and the source, provide a link to the Creative Commons license, and indicate if changes were made. The Creative Commons Public Domain Dedication waiver (http://creativecommons.org/publicdomain/zero/1.0/) applies to the data made available in this article, unless otherwise stated. 


\section{Background}

Nuclear pore complexes (NPCs) are channels that mediate bidirectional trafficking between the cytoplasm and the nucleus in eukaryotic cells. In vertebrates, multiple copies of approximately 30 polypeptides, termed nucleoporins (Nups), assemble to form NPCs [1, 2]. A single NPC exhibits an eight-fold symmetric cylinder-like structure that can be divided into three distinct regions $[2,3]$. One such region is the central channel, which spans the inner and outer nuclear membrane. The other two regions are the nuclear basket and the cytoplasmic filaments, both of which are joined by the central channel. Approximately one-third of nucleoporins have a variety of phenylalanine-glycine (FG) repeats [3, 4]. FG repeat-containing Nups form unstructured barriers in the central channel, which are crucial for the selective trafficking of macromolecules [4, 5].

Nup88 is a non-FG Nup located on the cytoplasmic face of the NPC that participates in nuclear export by interacting with other FG Nups, such as Nup358 and Nup214 [3, 6-8]. Intriguingly, abnormally high levels of Nup88 has been detected in the cytoplasm of various tumor cells [3, 9, 10]. Furthermore, it has been reported that elevated Nup88 expression in tumor cells is correlated with tumor grade such as in colorectal cancer, breast cancer and hepatocellular carcinoma [11-15]. Consequently, it has been proposed that Nup88 might be used as a cancer prognostic and diagnostic marker [3].

Recent reports indicate that Nup88 overexpression is responsible, at least in part, for the early steps of tumorigenesis. For instance, overexpression of Nup88 in HeLa cells induces multipolar spindles and the appearance of multinucleated cells [16]. In addition, elevated Nup88 expression in a mouse model induces multinuclei by promoting the premitotic destruction of Polo-like kinase 1 (Plk1), a kinase required for bipolar spindle formation and chromosome segregation $[17,18]$. However, the molecular basis of how Nup88 leads to a multinucleated phenotype is poorly understood because of a lack of information concerning its interacting partners [19].

Proteomic analysis reveals a variety of proteins that associate with NPCs [1]. One such protein is vimentin, which is classified as a type III intermediate filament (IF) protein abundant in motile mesenchymal cells, various types of tumors, and cancer cell lines [20]. Vimentin forms insoluble filamentous networks that distribute widely in the cytoplasm from the perinuclear region to the cell periphery. Phosphorylation of vimentin turns its insoluble filaments into a soluble or insoluble nonfilamentous form [21-23]. This organizational change of vimentin endows the cells with mechanical resistance, allowing them to undergo morphological change and motility [20, 24-27].
Proteomic approaches indicate that the N-terminal region of vimentin contains many amino acid (aa) residues that are phosphorylated by a variety of kinases, such as mitotic kinases [20, 25, 28, 29]. For instance, phosphorylation by cyclin-dependent kinase 1 (Cdk1) promotes vimentin IF disassembly into non-filamentous oligomers. In addition, Cdk1 promotes Plk1 specific vimentin phosphorylation during early mitosis [30-32]. Both Rhoassociated kinase and Aurora B kinase promote the segregation of vimentin IF cooperatively at the cleaved furrow from late mitosis to cytokinesis [30, 33, 34]. Although vimentin overexpression in cancer cells has been reported to be closely correlated with accelerated tumor growth, migration, and invasion, specific regulation of the phosphorylation status of vimentin during the development of cancer remains poorly understood [20].

In the present study, to gain insights into the molecular basis of how overexpressed Nup88 induces multinuclei in HeLa cells, we screened Nup88 interacting proteins using a proteomic approach. Here, we demonstrate that a novel interaction of Nup88 with vimentin can affect the phosphorylation status of vimentin. We also propose that the interaction between Nup88 and vimentin is involved in the Nup88-dependent multinucleated phenotype.

\section{Methods}

\section{Plasmid constructs}

Plasmids expressing GFP-tagged proteins under the control of a CMV promoter were generated by cloning their PCR products into pEGFP-N2 (Clontech, Mountain View, CA). The expression plasmids for FLAG-tagged proteins were constructed by replacing the EGFP genes in pEGFP-N2 with synthetic oligonucleotides encoding 3 x FLAG. A plasmid that expresses GST-fused Nup88 was constructed by inserting the Nup88 gene downstream of the GST gene in pGEX4T-3 (GE Healthcare Life Sciences, Piscataway, NJ). A plasmid that expresses Halo tagged Nup214 or Nup358 was purchased from Kazusa Genome Technologies Inc. [35].

\section{Cell culture and stable cell lines}

Cells were cultured in Dulbecco's modified Eagle's medium (DMEM) supplemented with $10 \%$ fetal bovine serum and penicillin/streptomycin at $37{ }^{\circ} \mathrm{C}$ in a humidified incubator supplied with $5 \% \mathrm{CO}_{2}$.

T-REx HeLa cells containing a single Flp recombination site in the genome were established from HeLa R19 cells [36]. The T-REx HeLa stable cell lines were established according to the manufacturer's instructions (Life Technologies, Carlsbad, CA). Briefly, the T-REx HeLa cells were cotransfected with pcDNA5/FRT/TO encoding an EGFP-tagged version of the genes of interest and pOG44 encoding the Flp recombinase. Following 
transfection, the cells were transferred into medium containing $100 \mu \mathrm{g} / \mathrm{ml}$ hygromycin B and $3 \mu \mathrm{g} / \mathrm{ml}$ blasticidin $\mathrm{S}$ to select for drug-resistant clones. The clones were collected by trypsinization and stored frozen in liquid $\mathrm{N}_{2}$ before use. Protein expression in T-REx HeLa cells was induced using medium containing $1 \mu \mathrm{g} / \mathrm{ml}$ doxycycline (DOX) for 24-48 h.

\section{GFP-coprecipitation assay}

Coimmunoprecipitation assays for GFP-fused proteins were performed as previously described [37]. Cell lysates (500-1500 $\mu \mathrm{g})$ prepared with NP-40 lysis buffer $(10 \mathrm{mM}$ Tris/ $\mathrm{HCl} \mathrm{pH} 7.5,150 \mathrm{mM} \mathrm{NaCl}, 5 \mathrm{mM}$ EDTA, 0.5\% Nonidet P-40) were incubated with 5-20 $\mu \mathrm{l}$ of GFP-trap A beads (ChromoTek, Munich, Germany) at $4{ }^{\circ} \mathrm{C}$ for 15-120 min. After incubation, the beads were collected by centrifugation at $2000 \mathrm{rpm}$ at $4{ }^{\circ} \mathrm{C}$ for $1 \mathrm{~min}$ and washed three times with lysis buffer. The proteins on the beads were eluted by incubation with $2.5 \times$ SDS sample buffer $(0.156 \mathrm{M}$ Tris/HCl pH 6.8, 5\% SDS, 25\% glycerol, $0.0125 \%$ bromophenol blue, $12.5 \%$ 2-mercaptoethanol) at $100{ }^{\circ} \mathrm{C}$ for $1 \mathrm{~min}$ and then analyzed by SDS-PAGE. Protein bands were visualized by staining with Coomassie brilliant blue (CBB). Proteins extracted from the gel were identified by mass analysis using matrix-assisted laser desorption time-of-flight mass spectrometry.

\section{Purification of GST fusion proteins}

E. coli cells expressing GST and GST fusion protein were harvested by centrifugation. The cell pellet was resuspended in NP-40 lysis buffer supplemented with $1 \%$ Triton X-100 and appropriate protease inhibitors. Cells were lysed by sonication. The supernatant was collected after centrifugation and incubated with glutathioneSepharose $4 \mathrm{~B}$ beads at $4{ }^{\circ} \mathrm{C}$ for 120 min with rotation. Proteins bound to the beads were washed three times with NP-40 lysis buffer and then eluted with elution buffer $(5 \%$ glycerol, $50 \mathrm{mM}$ Tris- $\mathrm{HCl} \mathrm{pH} 8.0,20 \mathrm{mM}$ reduced glutathione, $0.5 \%$ NP-40, $200 \mathrm{mM} \mathrm{NaCl}, 2.5 \mathrm{mM}$ $\mathrm{MgCl}_{2}, 1 \mathrm{mM}$ PMSF, $0.1 \mathrm{mM}$ dithiothreitol). Eluates were dialyzed overnight against NP-40 lysis buffer supplemented with appropriate protease inhibitors at $4{ }^{\circ} \mathrm{C}$.

\section{GST-pulldown assay}

Purified GST fusion protein was incubated with recombinant human vimentin purified from E. coli (PeproTech, Rocky Hill NJ) and glutathione Sepharose 4B beads in NP-40 lysis buffer at $4{ }^{\circ} \mathrm{C}$ for 120 min with rotation. The beads were washed three times with NP-40 lysis buffer. Proteins bound to the beads were eluted with $2.5 \times$ SDS sample buffer.

\section{Antibodies}

Mouse monoclonal anti-human Nup88 (\#611896) was purchased from BD Biosciences (Franklin Lakes, NJ). Rabbit polyclonal anti-GFP (\#598), anti- $\alpha$ tubulin (\#PM054), and anti-DDDDK (\#PM020) were purchased from Medical \& Biological Laboratories (Tokyo, Japan). Rabbit polyclonal anti-GFP (\#ab290), mouse monoclonal anti-vimentin (\#sc6260), rabbit monoclonal anti-pS83 vimentin (\#12569), rabbit polyclonal anti-phospho-Histone $\mathrm{H} 3$ (\#06-570), and mouse monoclonal anti-HaloTag (\#G9211) were purchased from Abcam (Cambridge, UK), Santa Cruz Biotechnologies (Dallas, TX), Cell Signaling Technologies (Danvers MA), Merck Millipore (Burlington, MA), and Promega (Madison, WI), respectively. For immunoblotting, all antibodies were applied at a dilution of 1:1000. Antibodies were diluted according to the manufacturer's instructions for indirect immunofluorescence.

\section{Immunoblotting}

Cell lysates were prepared with either radio immunoprecipitation assay (RIPA) buffer $(50 \mathrm{mM}$ Tris/ $\mathrm{HCl} \mathrm{pH} \mathrm{8.0,}$ $150 \mathrm{mM} \mathrm{NaCl}, 5 \mathrm{mM}$ EDTA, 1\% Nonidet P-40, 0.5\% sodium deoxycholate, $0.1 \%$ SDS) or NP-40 lysis buffer supplemented with appropriate protease inhibitors. Lysates were subjected to SDS-PAGE and proteins separated on the gel were transferred onto an Immobilon-P transfer membrane (Merck Millipore). The membrane was blocked with blocking buffer (5\% skimmed milk, 0 . 05\% Tween 20 in PBS) for $30 \mathrm{~min}$ at room temperature and then incubated with primary antibodies (diluted with $1 \% w / v$ skimmed milk, $0.05 \%$ Tween 20 in PBS) at $4{ }^{\circ} \mathrm{C}$ for more than $10 \mathrm{~h}$. After incubation, the membrane was washed with wash buffer $(0.05 \%$ Tween 20 in PBS) three times at $5 \mathrm{~min}$ intervals and then incubated with secondary antibodies conjugated with horseradish peroxidase (HRP) at $4{ }^{\circ} \mathrm{C}$ for $1 \mathrm{~h}$. Chemiluminescence was generated by the addition of Luminate Crescendo Western HRP substrate (Merck Millipore) to the membrane and was detected using a LAS2000 Imaging analyzer (Fuji Film, Tokyo, Japan).

\section{siRNA transfection}

Control siRNA and vimentin specific siRNA (\#4427038) were purchased from Ambion (Foster City, CA). The transfection of siRNA was performed using RNAiMAX transfection reagent (Life Technologies). Briefly, 1 day before transfection, cells were plated at a density of $4 \times$ $10^{5}$ cells per well in a 12-well plate with $1 \mathrm{ml}$ of culture medium and then cultured until $80 \%$ confluent. siRNA was then incubated with the transfection reagent in a reduced serum medium for 25 min to form a lipid-siRNA complex, and then transfected into cells. After a $24 \mathrm{~h}$ incubation, the medium was exchanged with fresh pre- 
warmed medium. The cells were further incubated until immediately prior to analyses.

\section{Indirect immunofluorescence assay}

Cells grown on coverslips in 24-well plates were washed once with pre-warmed PBS and then fixed with $4 \%$ paraformaldehyde for $15 \mathrm{~min}$, followed by permeabilization with PBS containing $0.5 \%$ Triton X-100 for $15 \mathrm{~min}$ at room temperature. The cells were then incubated with blocking buffer (3\% bovine serum albumin, $0.05 \%$ Triton $\mathrm{X}-100$ in PBS) for $1 \mathrm{~h}$ and then reacted with primary antibodies diluted in blocking buffer for $1 \mathrm{~h}$ at room temperature. After reaction, excess primary antibodies were removed by immersing the coverslips in wash buffer (1.5\% bovine serum albumin, $0.05 \%$ Triton X-100 in PBS) three times at $5 \mathrm{~min}$ intervals. Secondary antibodies conjugated with Alexa Fluor 568 or 488 (Thermo Fisher) were added to the cells, which were incubated in the dark for $1 \mathrm{~h}$ at room temperature. The cells were then soaked in the wash buffer three times at 5 min intervals and incubated with PBS containing Hoechst 33342 for 5 min at room temperature to stain the nuclei. Coverslips were mounted using Prolong Gold antifade reagent (Life Technologies). Fluorescence was detected using an ECLIPSE TE2000-U inverted microscope (Nikon, Tokyo, Japan).

\section{Results}

Nup88 interacts with vimentin intermediate filament protein

We attempted to establish stable cell lines expressing either GFP or GFP-tagged Nup88 (Nup88-GFP) as materials for screening Nup88 interacting proteins and for subsequent experiments. Because overexpression of Nup88 in HeLa cells is reported to induce multipolar spindles and the appearance of multinucleated cells [16], a tet-on expression system was applied in order to reduce both cell death and unexpected defects caused by Nup88 overexpression during the development of the stable cell lines. As shown in Fig. 1a, Nup88-GFP expression was undetectable in the absence of doxycycline (DOX). By contrast, GFP expression was strongly induced in the presence of DOX, indicating that the tet-on system worked well (Fig. 1a, lanes 3 and 4). When overexpressed, Nup88-GFP was localized to the nuclear rim and in the cytoplasm (Fig. 1b). A small portion of the protein was also found in the nucleus (Fig. 1b). Furthermore, overexpression of Nup88 induced multiple nuclei (Fig. 4b and c). These observations were consistent with previous reports $[16,19]$.

To screen Nup88 interacting proteins, we subjected the established cell lines to a GFP-coprecipitation assay. Cells expressing either GFP or Nup88-GFP upon DOX addition were extracted with NP-40 lysis buffer, and resultant lysates were incubated with anti-GFP antibody-conjugated agarose beads. Proteins recovered from the beads were separated by SDS-polyacrylamide gel electrophoresis and visualized with CBB (Fig. 2a). Proteins specifically recovered from Nup88-GFP bound beads were then subjected to mass spectrometry. As a result, vimentin, a type III IF protein, was obtained as a candidate Nup88 interacting protein (Fig. 2a lane 3). We then confirmed this interaction by an immunoblotting experiment using a vimentin
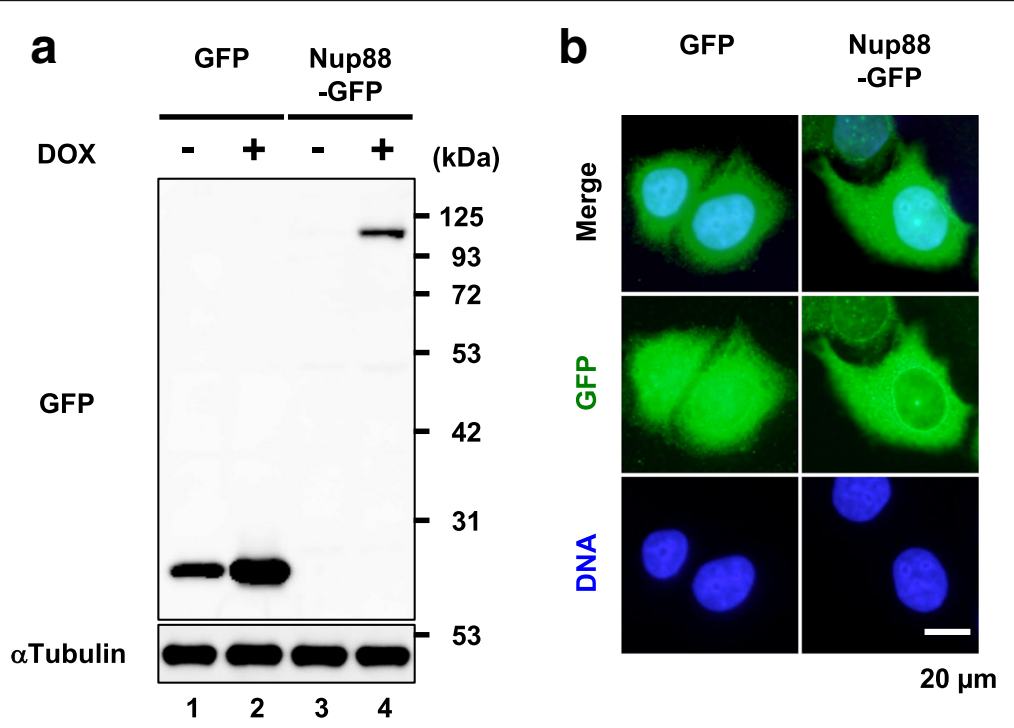

Fig. 1 Overexpression of GFP-tagged Nup88 in HeLa cells. Stable transfectants harboring genes encoding GFP or GFP-fused NUP88 under the control of a tetracycline-inducible system were incubated in medium without (-) or with (+) DOX for 2 days to stimulate protein overexpression. Overexpression and distribution pattern of the proteins in cells were assessed by immunoblotting (a) and by indirect immunofluorescence analysis (b), respectively 


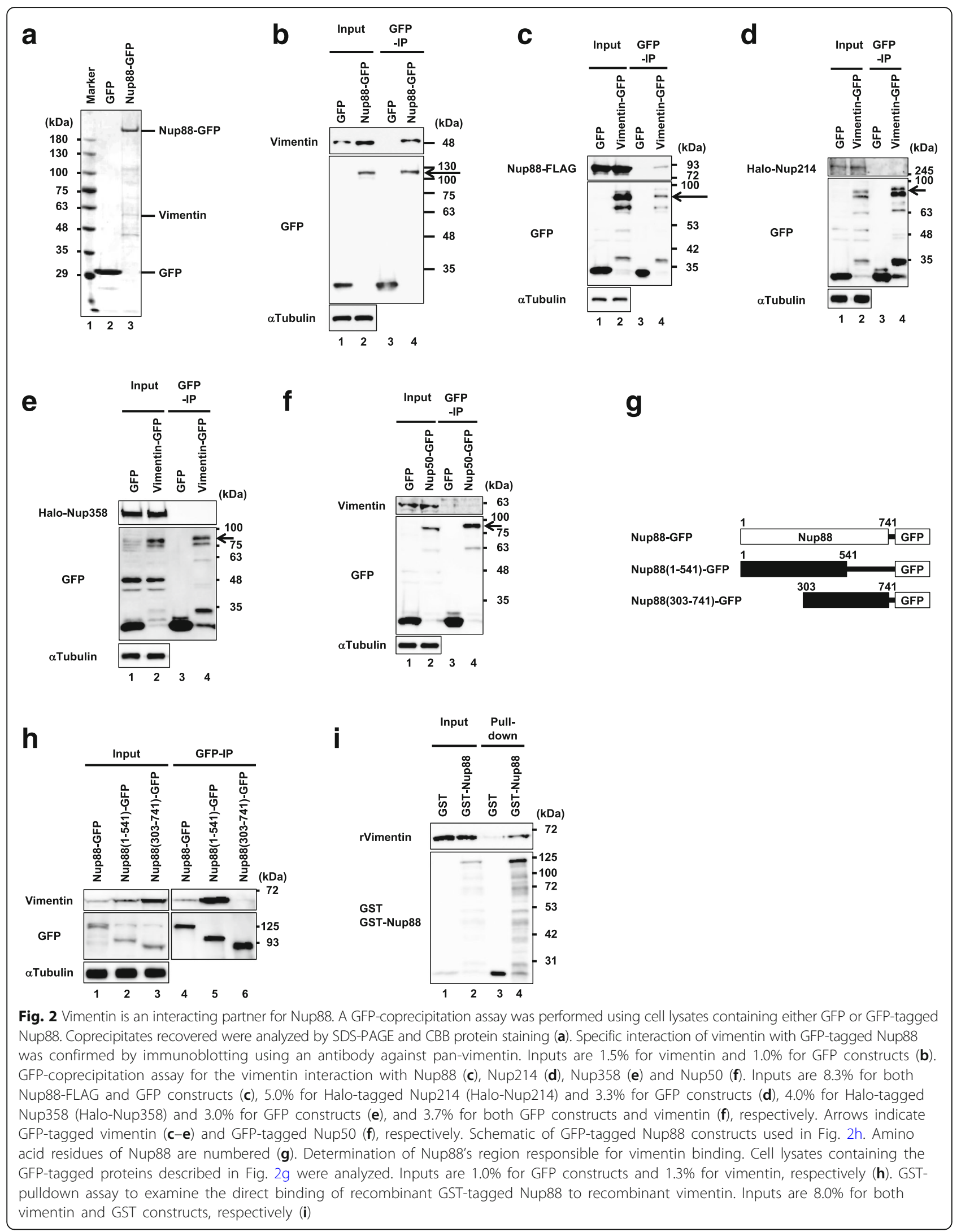


specific antibody (Fig. 2b). In addition, the interaction was reproduced when lysates containing GFP-tagged vimentin (Vimentin-GFP) and FLAG-tagged Nup88 (Nup88-FLAG) were employed in the GFP-coprecipitation assay (Fig. 2c).

To address the specificity of vimentin binding to Nup88, we examined its binding to Nup214, Nup358, and Nup50. Nup214, which is localized on the cytoplasmic face of the NPC and forms a subcomplex with Nup88 [6], did not coprecipitate with Vimentin-GFP (Fig. 2d). Similarly, Nup358, which is also on the cytoplasmic face of NPCs and interacts with Nup88 [7], did not coprecipitate (Fig. 2e). Moreover, we found no evidence that Nup50, a component of the nuclear basket of NPC [38], interacts with Vimentin-GFP (Fig. 2f). Taken together, these experiments indicated vimentin-binding to Nups is specific for Nup88.

To determine the region of Nup88 responsible for vimentin binding, we generated GFP-tagged $\mathrm{N}$ - or Cterminal fragments of Nup88 for use in a GFPcoprecipitation assay (Fig. 2g). Coprecipitation of Nup88-GFP and vimentin was observed (Fig. 2h lane 4). Moreover, the N-terminal 541 aa residues of Nup88 (Nup88(1-541)-GFP) showed clear coprecipitation of vimentin, whereas the corresponding C-terminal 439 aa residues (Nup88(303-741)-GFP) did not (Fig. 2h lanes 4 and 5). These results suggested that the region of Nup88 spanning at least from 303 to 541 aa residues is involved in vimentin binding.

It has been reported that Nup88 does not bind to vimentin directly in an assay using purified recombinant proteins [39]. Given that we observed binding between Nup88 and vimentin using HeLa cell lysates, we reasoned the interaction might be indirect i.e., mediated by other proteins. To examine whether the interaction is indirect or not, we performed a GST-pull down assay using purified recombinant GST-tagged Nup88 (GSTNup88) and recombinant human vimentin, both of which were prepared from $E$. coli (Fig. 2i). In this assay, recombinant vimentin was incubated with GST or GSTNup88 in the presence of glutathione-conjugated Sepharose beads, and the proteins recovered from the beads were then analyzed by an immunoblotting assay. The results of the experiments showed GST coprecipitated vimentin at a much reduced level, while GST-Nup88 coprecipitated at a much higher level than GST (Fig. 2i lanes 3 and 4). These observations indicated that the interaction between Nup88 and vimentin is direct in vitro.

Vimentin is functionally associated with the formation of multiple nuclei caused by Nup88 overexpression

In order to examine the in vivo association of Nup88 with vimentin, an indirect immunofluorescence assay was performed (Fig. 3). Overexpressed Nup88-GFP was clearly observed at the nuclear rim and in the cytoplasm

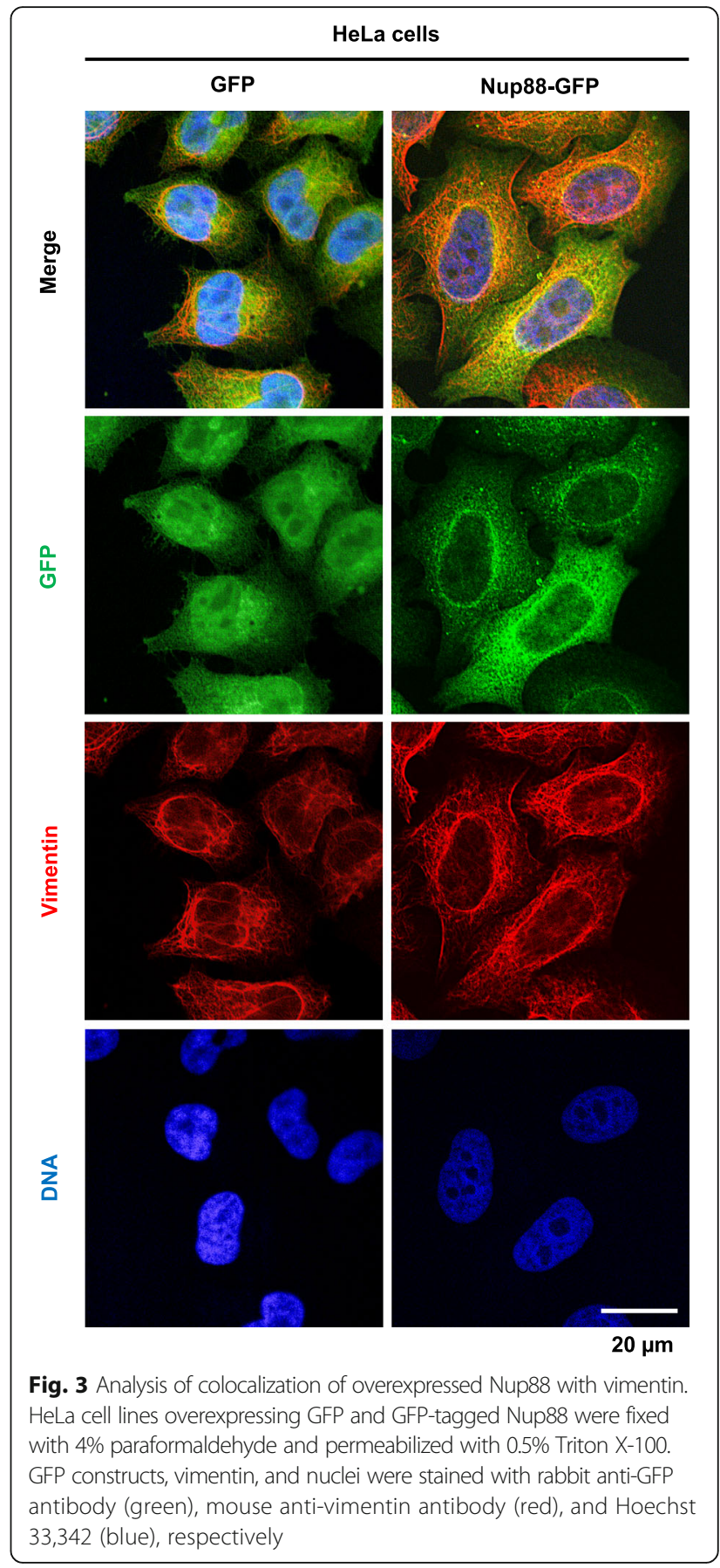

and to a lesser extent in the nucleus. By contrast, vimentin was observed exclusively in the cytoplasm. Because overexpressed Nup88 in tumor tissues is observed in the cytoplasm [3], we anticipated that Nup88 would colocalize with vimentin in the cytoplasm. However, colocalization of Nup88-GFP and vimentin in the cytoplasm was unclear due to the widespread diffusion of Nup88-GFP, while evidence of colocalization was seen at or around the nuclear rim (Fig. 3). We therefore switched the experiment to analyze the functional characterization of the 
interaction on Nup88-dependent multinuclei formation by vimentin depletion (Fig. 4). An immunoblotting assay revealed that vimentin specific siRNA transfection resulted in an approximately $70 \%$ reduction of vimentin expression without affecting the endogenous expression of Nup88 in both GFP and Nup88-GFP expressing cells (Fig. 4a). An indirect immunofluorescence assay also confirmed the depletion of vimentin (Fig. 4b). We then examined the effect of vimentin depletion on multiple nuclei formation by counting cells containing multinuclei (Fig. 4b). Control transfectants expressing GFP or Nup88-GFP showed the proportion of multinucleated cells to be 7.5 and $18.2 \%$, respectively, while vimentin siRNA transfectants of these cell lines showed the proportion of multinucleated cells to be 5.7 and $9.8 \%$, respectively. These results suggested a functional association between vimentin and the formation of multinuclei.

\section{Nup88 protects phospho-vimentin from dephosphorylation through interacting with the $\mathrm{N}$ - terminal region of vimentin}

The phosphorylation status of vimentin is critical for its organization and function [20]. We therefore examined
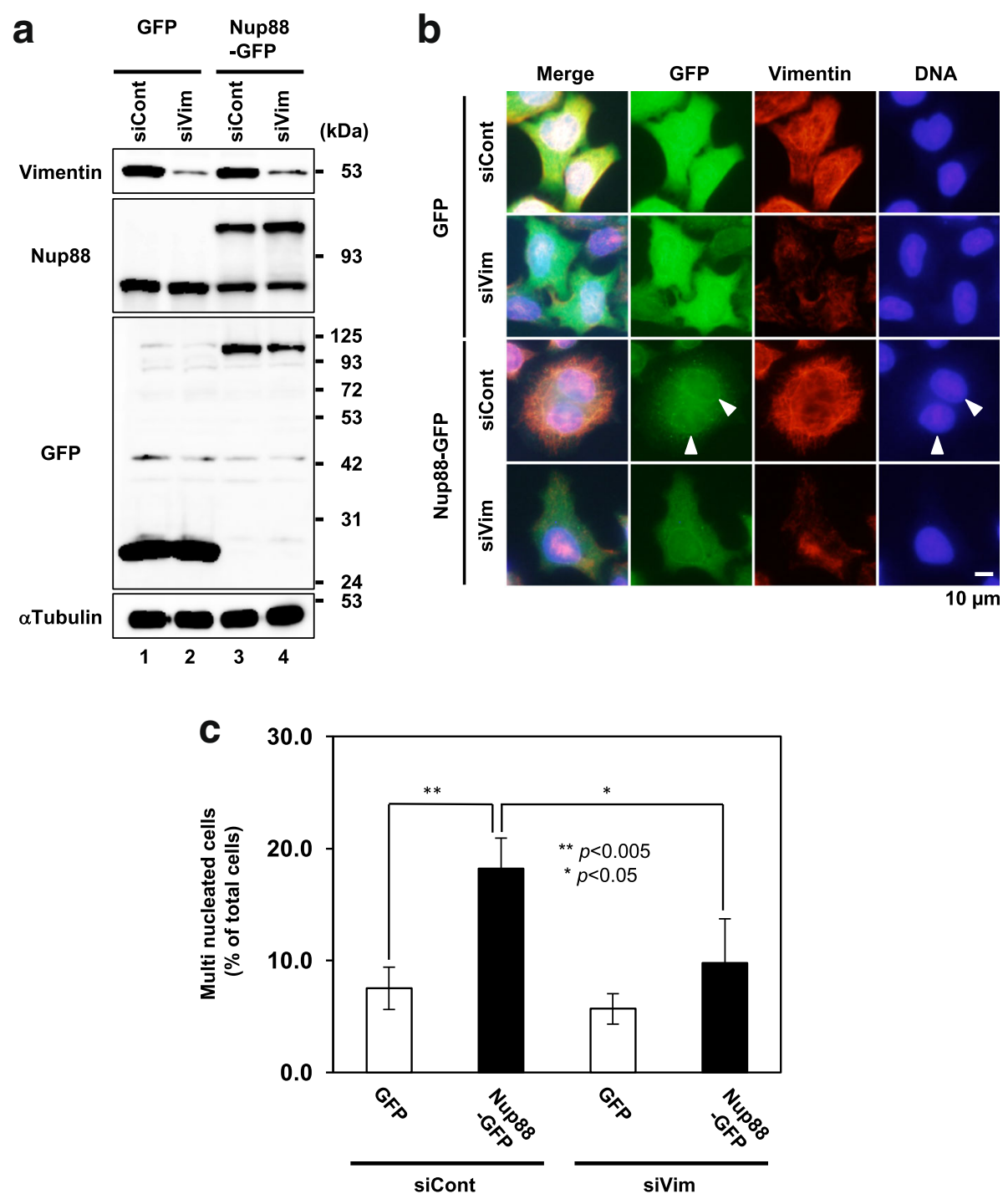

Fig. 4 Depletion of vimentin by siRNA transfection suppresses the formation of multinuclei induced by Nup88 overexpression. HeLa cell lines treated with control siRNA (siCont) or vimentin-specific siRNA (siVim) were overexpressed with GFP and GFP-tagged Nup88 in the presence of DOX for 2 days. Depletion of vimentin was confirmed by immunoblotting (a) and immunofluorescence (b). Multinucleated cells were counted in images obtained from the indirect immunofluorescence assay. Data were collected from at least four independent experiments. Total cell number analyzed in the experiment for siCont-treated GFP expressing cells, siCont-treated Nup88-GFP expressing cells, siVim-treated GFP expressing cells, and siVim-treated Nup88-GFP expressing cells were 1296, 1156, 1377 and 1284 cells, respectively (c). White arrowheads indicate multinuclei (binuclei) (b). Error bars indicate $\pm \mathrm{SD}(\mathbf{c})$ 
the phosphorylation status of vimentin by monitoring phospho-Ser83 (pS83), which is phosphorylated by Plk1 during mitosis [30]. The phosphorylation of Ser83 in Nup88-GFP expressing cells was approximately 2-fold higher than that in GFP expressing cells, even though total vimentin expression was comparable between these cell lines (Fig. 5a and b). It is reported that vimentin IF is phosphorylated predominantly in mitosis [30-32] and that overexpression of Nup88 induces a mitotic defect in HeLa cells [16]. We therefore considered the possibility that the increased level of pSer83 is due to cell cycle
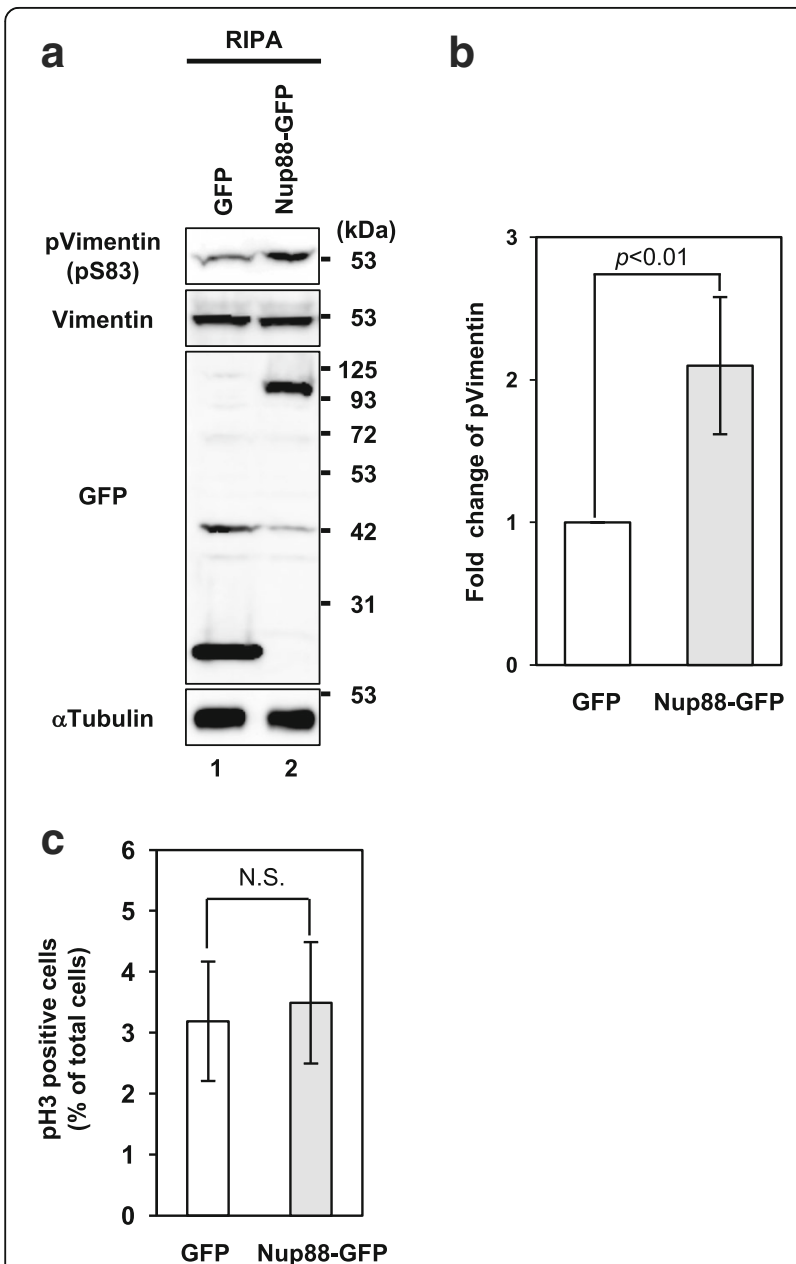

Fig. 5 Increased expression of phospho-vimentin in Nup88 overexpressing cells. Phospho-vimentin expression was analyzed in HeLa cells overexpressing GFP and Nup88-GFP by immunoblotting using antibodies against pan-vimentin, phospho-vimentin (pS83), GFP and a-tubulin (a). Relative expression of phospho vimentin in cells expressing GFP and Nup88-GFP (b). Data were collected from three independent experiments including the result shown in Fig. 5 a (b). Cells in mitosis were monitored by an immunofluorescence assay. HeLa cells overexpressing GFP or GFP-tagged Nup88 were fixed and stained with antibodies against phospho-histone $\mathrm{H3}(\mathrm{pH} 3)$ to distinguish mitotic cells from interphase cells. Data were collected from six independent experiments. Error bars indicate \pm SD (b and $\mathbf{c})$. N. S. means not significant (c) delay or arrest in $\mathrm{M}$ phase. To estimate the ratio of cells in $\mathrm{M}$ phase, an indirect immunofluorescence assay using anti-phospho-Histone $\mathrm{H} 3$ was performed. As shown in Fig. 5c, the ratio was similar between GFP and Nup88GFP expressing cells. Thus, these results indicated that the increase of phospho-vimentin in Nup88-GFP expressing cells was not due to arrest or delay of $\mathrm{M}$ phase.

The results shown in Fig. 5 raised the question of how Nup88 increases the level of phospho-vimentin. Because Nup88 was found to bind to vimentin directly (Fig. 2i), we considered two possibilities for the observed increase in phospho-vimentin as follows; either (i) Nup88 recruits kinases to vimentin or (ii) the interaction with Nup88 sterically prevents vimentin from being dephosphorylated by phosphatases. To examine the latter possibility, we first prepared cell lysates from GFP and Nup88-GFP expressing cells arrested in metaphase by colcemid treatment (Fig. 6a) before confirming the abundance of phospho-vimentin (Fig. 6b lanes 1 and 2). We subsequently analyzed the effect of Nup88 on endogenous phosphatases to monitor the decreased levels of phospho-vimentin. By comparison to the 0 min control (Fig. 6b lanes 1 and 2), phospho-vimentin levels were reduced by incubation for $10 \mathrm{~min}$ in either cell lysate (Fig. $6 \mathrm{~b}$ lanes 3 and 4), suggesting that endogenous phosphatases were active under these experimental conditions. In addition, statistical analysis revealed that the decrease of phospho-vimentin in lysates containing Nup88-GFP was significantly lower than in lysates containing GFP (Fig. 6c). To further examine whether the inhibitory effect was specific to endogenous phosphatases or not, we assessed the phosphorylation status of vimentin in the presence of several units of bacteriophage lambda protein phosphatase ( $\lambda$ PPase). When cell lysates were incubated with $\lambda$ PPase, phospho-vimentin levels decreased in a manner dependent on $\lambda$ PPase activity (Fig. 6b, lanes 512). Phospho-vimentin in lysates containing Nup88-GFP was significantly more resistant to $\lambda$ PPase than in lysates containing GFP (Fig. 6d). These findings suggest that the protection of phospho-vimentin by Nup 88 is nonspecific to phosphatases.

There are a large number of phosphorylation sites located on the N-terminal 96 aa residues of vimentin [28, 29]. Because Nup 88 protects vimentin from phosphatases (Fig. 6), we speculated that the interaction site for Nup88 is within the $\mathrm{N}$-terminal region of vimentin. To examine this possibility, we performed a GFP-coprecipitation assay using lysates containing either full-length vimentin (VimFLAG) or a truncated version of the protein where the $\mathrm{N}$ terminal 96 aa residues were missing ( $\Delta 1-96-$ FLAG) (Fig. 7a). Both constructs were expressed at similar levels in Nup88-GFP expressing cells (Fig. 7b lanes 1 and 2). Although Nup88-GFP was precipitated at similar levels in both cell lysates, coprecipitation of Vim-FLAG was 2.6- 


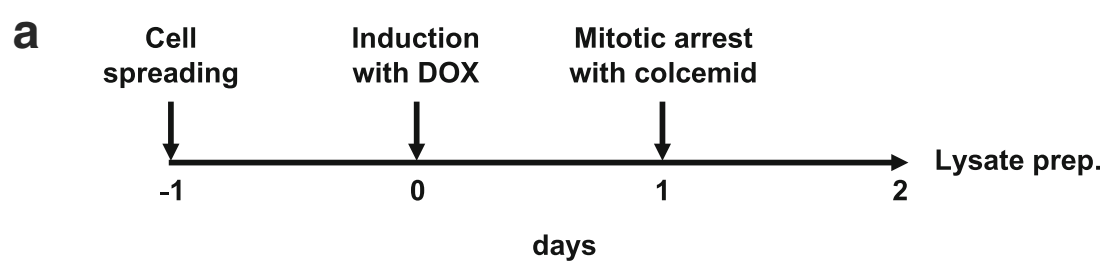

b
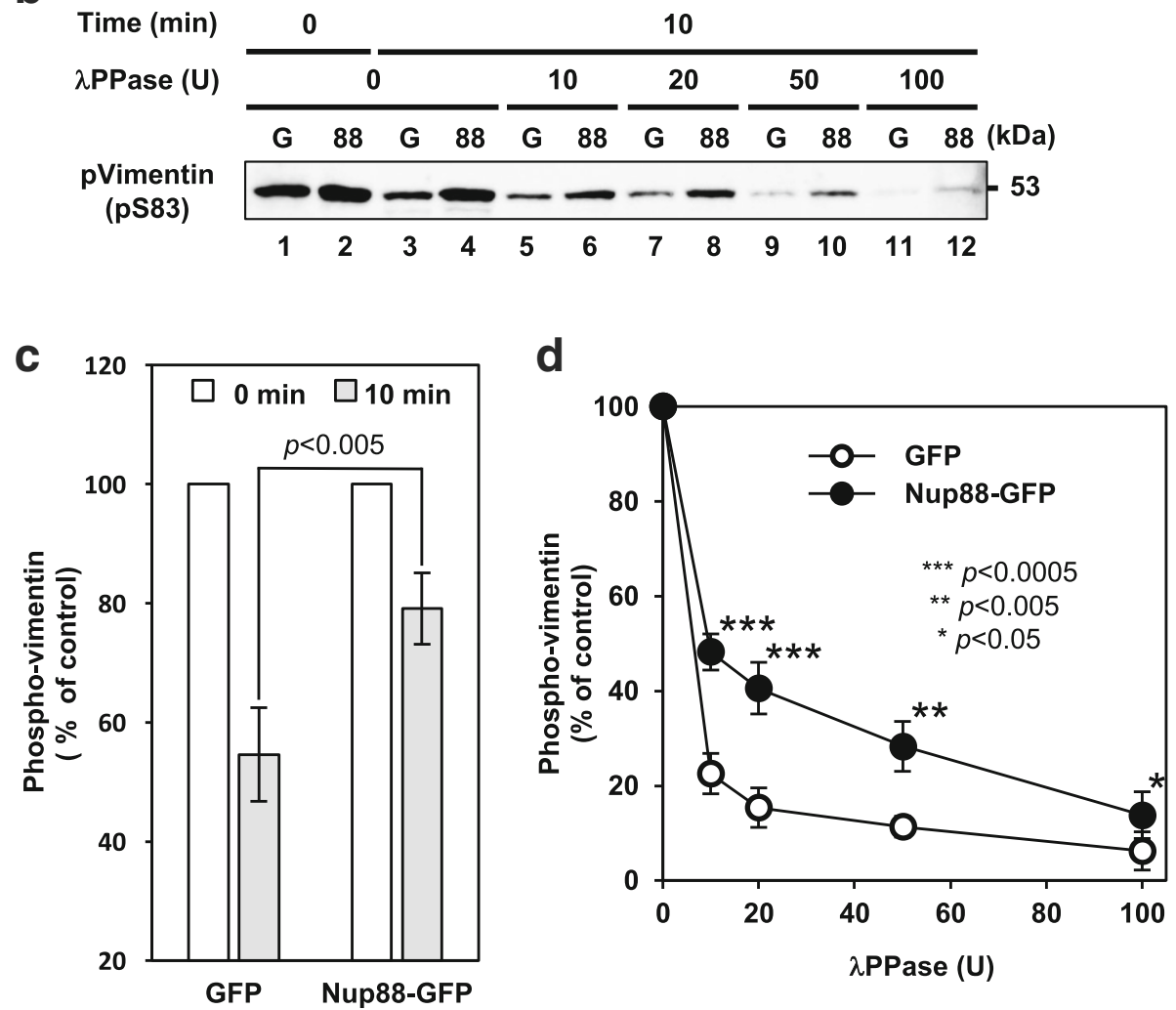

Fig. 6 Overexpression of Nup88 inhibits vimentin dephosphorylation. Schematic of cell lysates preparation from cells arrested in mitosis (a). Mitotic cell lysates containing GFP or Nup88-GFP were incubated in the absence and presence of $\lambda$ PPase for 10 min, followed by detecting phospho-vimentin (pVimentin) by immunoblotting assay (b). Decrease of pVimentin in the absence of $\lambda$ PPase corresponding to Fig. $6 \mathrm{~b}$ lanes 1-4 (c). A decrease of pVimentin in the presence of several units of $\lambda$ PPase corresponding to Fig. 6b lanes 3-12 (d). Data were collected from four independent experiments ( $\mathbf{c}$ and $\mathbf{d}$ ). Error bars indicate \pm SD of four experiments ( $\mathbf{c}$ and $\mathbf{d}$ )

fold higher than that of $\triangle 1$-96-FLAG (Fig. 7b lanes 3 and 4). These findings suggested the $\mathrm{N}$-terminal 96 aa residues of vimentin are required for its interaction with Nup88. To confirm its requirement more directly, either a GFPtagged N-terminal vimentin fragment (1-96-GFP) or control GFP was co-overexpressed with Nup88-FLAG in HeLa cells, and then the cell lysates were employed in the GFP-coprecipitation assay. As a result, 1-96-GFP specifically coprecipitated with Nup88-FLAG (Fig. 7c). We further confirmed the binding of 1-96-GFP to FLAG-tagged Nup88(1-541), which contains the vimentin binding region (Figs. $2 \mathrm{~h}$ and $7 \mathrm{~d}$ ). Taken together, these data indicated that the $\mathrm{N}$-terminal 96 aa residues of vimentin are responsible for Nup88 binding.

\section{Discussion}

Although recent studies have suggested that Nup88 overexpression can promote the early step of tumorigenesis by inducing formation of multinuclei, the molecular basis of how the multinucleated phenotype occurs is still poorly understood $[16,18]$. To better understand the molecular mechanism, we screened Nup88-interacting proteins by a proteomic approach and identified vimentin as a novel Nup88 interacting protein (Fig. 1). Nup88 was found to interact with the $\mathrm{N}$-terminal 96 aa residues of vimentin, which is a region of the protein predominantly phosphorylated in mitosis (Figs. 2 and 7). Moreover, this interaction with Nup88 protects phosphorylated vimentin from phosphatases (Fig. 4). Cells depleted in vimentin exhibited a 


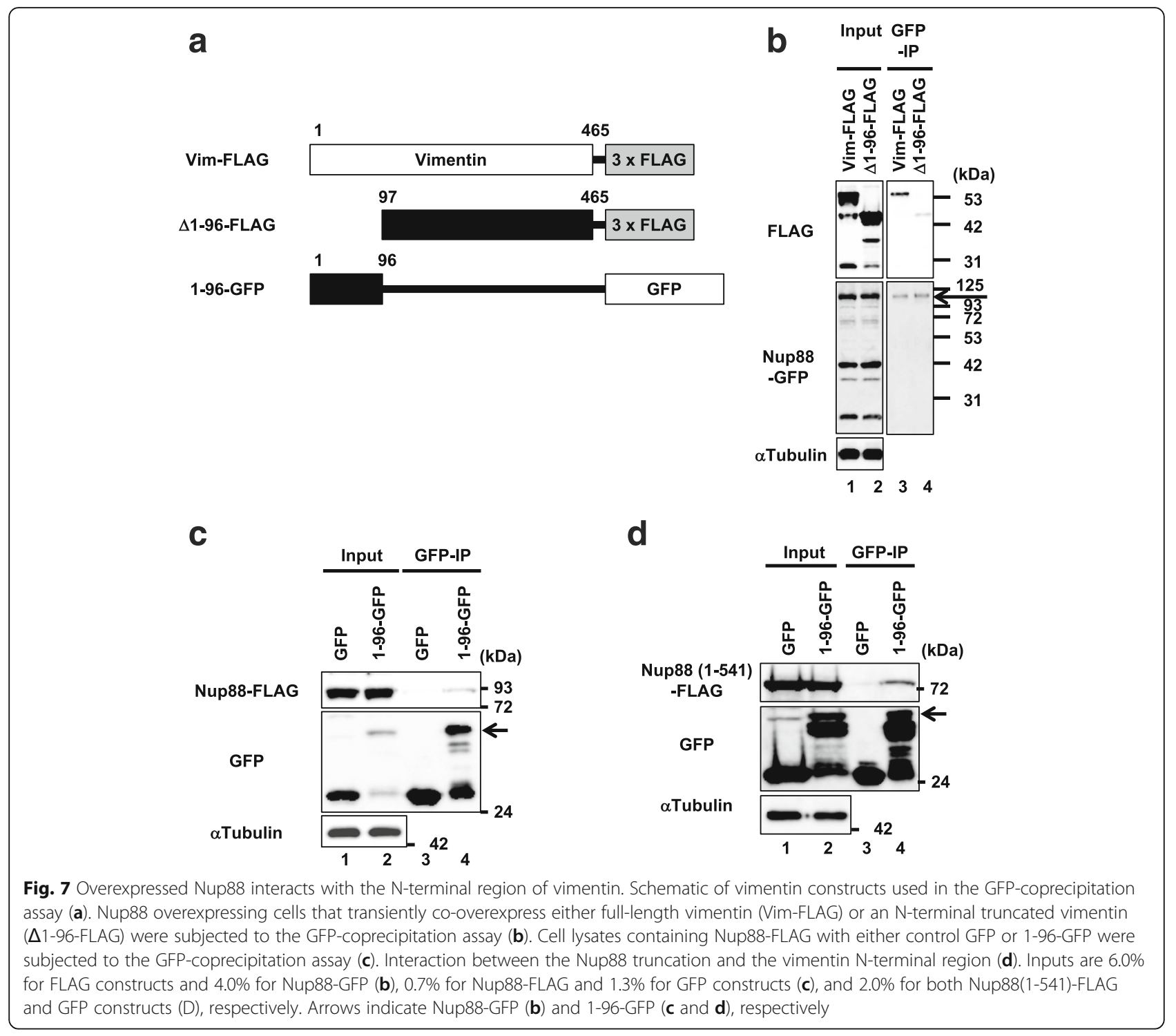

decreased occurrence of multinuclei caused by Nup 88 overexpression (Fig. 3).

It is reported that Nup88 binds to lamin A [39]. Interestingly, despite the fact that both lamin A and vimentin have a similar domain organization as IF proteins [23], lamin A is reported to bind to Nup88 through its Cterminal tail domain [39] whereas vimentin was found to interact with Nup88 through its $\mathrm{N}$-terminal region (Fig. 7). Nonetheless, these two IF proteins bind to almost the same region of Nup88 (Fig. 2). Our findings imply that the mode of binding of Nup88 to vimentin is distinct from that of Nup88 to lamin A.

We found that cells overexpressing Nup88 exhibited increased levels of phospho-Ser83 within the N-terminal 96 aa of vimentin (Fig. 5) and that dephosphorylation at the site occurs more gradually in mitotic lysates containing an excess amount of Nup88 than in control lysates
(Fig. 6). These results suggested that Nup88 inhibits phosphatases. Because the suppression was observed even in the presence of an unrelated phosphatase, it is unlikely that Nup88 actively and functionally inhibits such phosphatases (Fig. 6). Instead, rather than inhibiting the phosphatase directly, we propose a mechanism in which Nup88 masks the phosphorylated site and protects it from phosphatases. Moreover, our results show that Nup88 interacts with the $\mathrm{N}$-terminal region of vimentin (Fig. 7).

The phosphorylation status of vimentin is critical for its organization and function [20]. We found that the depletion of vimentin suppressed Nup88-dependent multinuclei formation (Fig. 4). However, it is still unclear how vimentin participates in the phenotype (Fig. 3). It is reported that both Ser71 and Ser72 within the Nterminal region of vimentin are phosphorylated by Rho- 
associated kinases and Aurora B, respectively [33, 34]. Mutant vimentin, in which Ser71 and Ser72 cannot be phosphorylated by these kinases, is also reported to give rise to multinuclei and aneuploidy [34, 40, 41]. In the present study, we found that Nup88 could bind to the $\mathrm{N}$-terminal region (Fig. 7) and protect phospho-Ser83 from phosphatases in HeLa cell lysates (Fig. 6). Moreover, a GST-pulldown assay using recombinant proteins purified from E. coli indicated that Nup88 could also bind to non-phosphorylated vimentin (Fig. 2i). It is therefore possible that Nup8 8 also masks the N-terminal region of vimentin against kinases as well as phosphatases. If this is the case, Nup88 may influence vimentin organization through protecting its $\mathrm{N}$-terminal region from kinases, thereby promoting the multinucleated phenotype.

The specific regulation of vimentin organization during cancer development has remained unclear even though vimentin expression is correlated with malignant transformation of cancer [20]. Here, we found that Nup88 could affect the phosphorylation status of vimentin (Fig. 6). Our results also suggest that Nup88 affects vimentin organization by altering its phosphorylation status, which contributes to cancer malignancy. To pursue this line of research, it would be informative to examine the co-overexpression of Nup88 with vimentin in malignant tumor tissues.

\section{Conclusions}

Nup88 can affect the phosphorylation status of vimentin, which may contribute to the Nup88-dependent multinucleated phenotype by changing the organization of vimentin.

\section{Abbreviations}

CMV: Cytomegalovirus; DOX: Doxycycline; GFP: Green fluorescent protein; IF: Intermediate filament; NP-40: Nonidet-P40; NPC: Nuclear pore complex; Nups: Nucleoporins; RIPA: Radio immunoprecipitation assay; siRNA: Small interference RNA

\section{Acknowledgments}

We thank Dr. Katharine Ullman (University of Utah) for providing HeLa cells and Dr. Shogo Misumi (Kumamoto University, Japan) for help with protein identification.

\section{Funding}

This work was supported by JSPS KAKENHI Grant Numbers JP24890288, JP26460087, and JP15K14983. The funder had no role in the conceptualization of the study design, data collection and analysis, or preparation of the manuscript.

\section{Availability of data and materials}

The raw data and materials generated in this study are available from the corresponding author upon reasonable request.

\section{Authors' contributions}

MM, $\mathrm{HN}$ designed and performed the experiments. MM, HN, AK interpreted the obtained results and wrote the manuscript. All authors read and approved the manuscript.

Ethics approval and consent to participate

No cell lines required ethics approval for their use.

\section{Competing interests}

The authors declare that they have no competing interests.

\section{Publisher's Note}

Springer Nature remains neutral with regard to jurisdictional claims in published maps and institutional affiliations.

Received: 26 July 2017 Accepted: 30 April 2018

Published online: 03 May 2018

\section{References}

1. Cronshaw JM, Krutchinsky AN, Zhang W, Chait BT, Matunis MJ. Proteomic analysis of the mammalian nuclear pore complex. J Cell Biol. 2002;158(5):915-27.

2. Jahed Z, Soheilypour M, Peyro M, Mofrad MR. The LINC and NPC relationship - it's complicated! J Cell Sci. 2016;129(17):3219-29.

3. Xu S, Powers MA. Nuclear pore proteins and cancer. Semin Cell Dev Biol. 2009;20(5):620-30

4. Alber F, Dokudovskaya S, Veenhoff LM, Zhang W, Kipper J, Devos D, Suprapto A, Karni-Schmidt O, Williams R, Chait BT, et al. The molecular architecture of the nuclear pore complex. Nature. 2007;450(7170):695-701.

5. Denning DP, Patel SS, Uversky V, Fink AL, Rexach M. Disorder in the nuclear pore complex: the FG repeat regions of nucleoporins are natively unfolded. Proc Natl Acad Sci U S A. 2003:100(5):2450-5.

6. Bernad R, Engelsma D, Sanderson H, Pickersgill H, Fornerod M. Nup214Nup88 nucleoporin subcomplex is required for CRM1-mediated $60 \mathrm{~S}$ preribosomal nuclear export. J Biol Chem. 2006;281(28):19378-86.

7. Bernad R, van der Velde $H$, Fornerod M, Pickersgill H. Nup358/RanBP2 attaches to the nuclear pore complex via association with Nup88 and Nup214/CAN and plays a supporting role in CRM1-mediated nuclear protein export. Mol Cell Biol. 2004;24(6):2373-84

8. Griffis ER, XU S, Powers MA. Nup98 localizes to both nuclear and cytoplasmic sides of the nuclear pore and binds to two distinct nucleoporin subcomplexes. Mol Biol Cell. 2003;14(2):600-10.

9. Gould VE, Martinez N, Orucevic A, Schneider J, Alonso A. A novel, nuclear pore-associated, widely distributed molecule overexpressed in oncogenesis and development. Am J Pathol. 2000:157(5):1605-13.

10. Martinez N, Alonso A, Moragues MD, Ponton J, Schneider J. The nuclear pore complex protein Nup88 is overexpressed in tumor cells. Cancer Res. 1999;59(21):5408-11.

11. Agudo D, Gomez-Esquer F, Martinez-Arribas F, Nunez-Villar MJ, Pollan M, Schneider J. Nup88 mRNA overexpression is associated with high aggressiveness of breast cancer. Int J Cancer. 2004;109(5):717-20.

12. Emterling A, Skoglund J, Arbman G, Schneider J, Evertsson S, Carstensen J, Zhang H, Sun XF. Clinicopathological significance of Nup88 expression in patients with colorectal cancer. Oncology. 2003:64(4):361-9.

13. Knoess M, Kurz AK, Goreva O, Bektas N, Breuhahn K, Odenthal M, Schirmacher P, Dienes HP, Bock CT, Zentgraf H, et al. Nucleoporin 88 expression in hepatitis $B$ and $C$ virus-related liver diseases. World J Gastroenterol. 2006;12(36):5870-4.

14. Schneider J, Martinez-Arribas F, Torrejon R. Nup88 expression is associated with myometrial invasion in endometrial carcinoma. Int J Gynecol Cancer. 2010;20(5):804-8.

15. Zhao ZR, Zhang LJ, Wang YY, Li F, Wang MW, Sun XF. Increased serum level of Nup88 protein is associated with the development of colorectal cancer. Med Oncol. 2012;29(3):1789-95.

16. Hashizume C, Nakano H, Yoshida K, Wong RW. Characterization of the role of the tumor marker Nup88 in mitosis. Mol Cancer. 2010;9:119.

17. Barr FA, Sillje HH, Nigg EA. Polo-like kinases and the orchestration of cell division. Nat Rev Mol Cell Biol. 2004;5(6):429-40.

18. Naylor RM, Jeganathan KB, Cao X, van Deursen JM. Nuclear pore protein NUP88 activates anaphase-promoting complex to promote aneuploidy. J Clin Invest. 2016;126(2):543-59.

19. Li J, Zhao J, Li Y. Multiple biological processes may be associated with tumorigenesis under NUP88-overexpressed condition. Genes Chromosomes Cancer. 2017;56(2):117-27.

20. Satelli A, Li S. Vimentin in cancer and its potential as a molecular target for cancer therapy. Cell Mol Life Sci. 2011;68(18):3033-46.

21. Eriksson JE, Brautigan DL, Vallee R, Olmsted J, Fujiki H, Goldman RD. Cytoskeletal integrity in interphase cells requires protein phosphatase activity. Proc Natl Acad Sci U S A. 1992;89(22):11093-7. 
22. Soellner P, Quinlan RA, Franke WW. Identification of a distinct soluble subunit of an intermediate filament protein: tetrameric vimentin from living cells. Proc Natl Acad Sci U S A. 1985;82(23):7929-33.

23. Herrmann H, Strelkov SV, Burkhard P, Aebi U. Intermediate filaments: primary determinants of cell architecture and plasticity. J Clin Invest. 2009; 119(7):1772-83.

24. Goldman RD, Khuon S, Chou YH, Opal P, Steinert PM. The function of intermediate filaments in cell shape and cytoskeletal integrity. J Cell Biol. 1996;134(4):971-83.

25. Ivaska J, Pallari HM, Nevo J, Eriksson JE. Novel functions of vimentin in cell adhesion, migration, and signaling. Exp Cell Res. 2007;313(10):2050-62

26. Mendez MG, Kojima S, Goldman RD. Vimentin induces changes in cell shape, motility, and adhesion during the epithelial to mesenchymal transition. FASEB J. 2010;24(6):1838-51.

27. Helfand BT, Mendez MG, Murthy SN, Shumaker DK, Grin B, Mahammad S, Aebi U, Wedig T, Wu YI, Hahn KM, et al. Vimentin organization modulates the formation of lamellipodia. Mol Biol Cell. 2011;22(8):1274-89.

28. Izawa I, Inagaki M. Regulatory mechanisms and functions of intermediate filaments: a study using site- and phosphorylation state-specific antibodies. Cancer Sci. 2006;97(3):167-74

29. Eriksson JE, He T, Trejo-Skalli AV, Harmala-Brasken AS, Hellman J, Chou YH, Goldman RD. Specific in vivo phosphorylation sites determine the assembly dynamics of vimentin intermediate filaments. J Cell Sci. 2004;117(Pt 6):919-32.

30. Yamaguchi T, Goto H, Yokoyama T, Sillje H, Hanisch A, Uldschmid A, Takai Y, Oguri T, Nigg EA, Inagaki M. Phosphorylation by Cdk1 induces Plk1-mediated vimentin phosphorylation during mitosis. J Cell Biol. 2005:171(3):431-6.

31. Chou YH, Bischoff JR, Beach D, Goldman RD. Intermediate filament reorganization during mitosis is mediated by p34cdc2 phosphorylation of vimentin. Cell. 1990;62(6):1063-71.

32. Tsujimura K, Ogawara M, Takeuchi Y, Imajoh-Ohmi S, Ha MH, Inagaki M. Visualization and function of vimentin phosphorylation by cdc2 kinase during mitosis. J Biol Chem. 1994;269(49):31097-106.

33. Goto H, Kosako H, Tanabe K, Yanagida M, Sakurai M, Amano M, Kaibuchi K, Inagaki M. Phosphorylation of vimentin by rho-associated kinase at a unique amino-terminal site that is specifically phosphorylated during cytokinesis. J Biol Chem. 1998;273(19):11728-36.

34. Goto H, Yasui Y, Kawajiri A, Nigg EA, Terada Y, Tatsuka M, Nagata K, Inagaki M. Aurora-B regulates the cleavage furrow-specific vimentin phosphorylation in the cytokinetic process. J Biol Chem. 2003;278(10):8526-30

35. Nagase T, Yamakawa H, Tadokoro S, Nakajima D, Inoue S, Yamaguchi K, Itokawa Y, Kikuno RF, Koga H, Ohara O. Exploration of human ORFeome: high-throughput preparation of ORF clones and efficient characterization of their protein products. DNA Res. 2008;15(3):137-49.

36. Kaiser C, Dobrikova EY, Bradrick SS, Shveygert M, Herbert JT, Gromeier M. Activation of cap-independent translation by variant eukaryotic initiation factor 4G in vivo. RNA. 2008;14(10):2170-82.

37. Makise M, Mackay DR, Elgort S, Shankaran SS, Adam SA, Ullman KS. The Nup153-Nup50 protein interface and its role in nuclear import. J Biol Chem. 2012;287(46):38515-22.

38. Guan T, Kehlenbach RH, Schirmer EC, Kehlenbach A, Fan F, Clurman BE, Arnheim N, Gerace L. Nup50, a nucleoplasmically oriented nucleoporin with a role in nuclear protein export. Mol Cell Biol. 2000;20(15):5619-30.

39. Lussi YC, Hugi I, Laurell E, Kutay U, Fahrenkrog B. The nucleoporin Nup88 is interacting with nuclear lamin A. Mol Biol Cell. 2011;22(7):1080-90.

40. Matsuyama M, Tanaka H, Inoko A, Goto H, Yonemura S, Kobori K, Hayashi Y, Kondo E, Itohara S, Izawa I, et al. Defect of mitotic vimentin phosphorylation causes microophthalmia and cataract via aneuploidy and senescence in lens epithelial cells. J Biol Chem. 2013;288(50):35626-35.

41. Tanaka H, Goto H, Inoko A, Makihara H, Enomoto A, Horimoto K, Matsuyama M, Kurita K, Izawa I, Inagaki M. Cytokinetic failure-induced Tetraploidy develops into aneuploidy, triggering skin aging in Phosphovimentindeficient mice. J Biol Chem. 2015;290(21):12984-98.

Ready to submit your research? Choose BMC and benefit from:

- fast, convenient online submission

- thorough peer review by experienced researchers in your field

- rapid publication on acceptance

- support for research data, including large and complex data types

- gold Open Access which fosters wider collaboration and increased citations

- maximum visibility for your research: over $100 \mathrm{M}$ website views per year

At BMC, research is always in progress.

Learn more biomedcentral.com/submissions 